\title{
Sea Ice Automatic Extraction in the Liaodong Bay from Sentinel- 2 Imagery Using Convolutional Neural Networks
}

\author{
Li Zherui, Cai Huiwen* \\ College of Marine Science and Technology, Zhejiang Ocean University, Zhoushan, 316000, China
}

\begin{abstract}
Sea ice classification is one of the important tasks of sea ice monitoring. Accurate extraction of sea ice types is of great significance on sea ice conditions assessment, smooth navigation and safty marine operations. Sentinel-2 is an optical satellite launched by the European Space Agency. High spatial resolution and wide range imaging provide powerful support for sea ice monitoring. However, traditional supervised classification method is difficult to achieve fine results for small sample features. In order to solve the problem, this paper proposed a sea ice extraction method based on deep learning and it was applied to Liaodong Bay in Bohai Sea, China. The convolutional neural network was used to extract and classify the feature of the image from Sentinel-2. The results showed that the overall accuracy of the algorithm was $85.79 \%$ which presented a significant improvement compared with the tranditional algorithms, such as minimum distance method, maximum likelihood method, Mahalanobis distance method, and support vector machine method. The method proposed in this paper, which combines convolutional neural networks and high-resolution multispectral data, provides a new idea for remote sensing monitoring of sea ice.
\end{abstract}

\section{Introduction}

Sea ice is an important part of the earth's climate, and it is one of the indicators of global climate change. Sea ice can affect marine hydro-logical conditions, atmospheric circulation, and ocean climate, and it is the most prominent marine disaster in middle and high latitudes sea areas $[1,2]$. Sea ice will bring direct losses to marine transportation, marine fisheries, and development of marine oil and gas resources. In recent years, the global climate is abnormal, the chances of sea ice appearing is more and more, which makes it difficult for ships to go through, making the sea operation more difficult, causing serious economic losses. The severe impact on marine fisheries, coastal construction industry and manufacturing industry is dramatic. Accurate identification of sea ice types is important on understanding the sea ice conditions, ensuring safe navigation and monitoring the climate change variation.

When sea ice occurs, it is hard for ships to reach and to have the monitoring work done. Traditional in situ methods such as ocean station observation and ice area survey are limited to time and space constraints, and also, it is difficult to meet the requirements of large-scale spatial information acquisition and dynamic monitoring of time series. Remote sensing is a technology that uses electromagnetic wave to detect and identify ground objects[3]. Remote sensing can get large-scale data more quickly and with high accuracy, and has become one of the important methods on sea ice monitoring and analysing. The remote sensing data used for sea ice usually comes from SAR(Synthetic Aperture Radar), MODIS(Moderate Resolution Imaging Spectrometer), Landsat and Hyperspectral images. Data from SAR were used for sea ice classification quite often in a lot of previous studies. Dabboor used RADARSAT Constellation Mission for parameter construction and optimization, and proposed a new sea ice classification parameter[4]. Ressel built a gray-level co-occurrence matrix based on TerraSAR-X data and proposed a framework for sea ice classification based on neural network $[5,6]$. Very limited study use high-resolution multispectral satellites like Sentinel-2 to classify the sea ice.

Deep learning is a new wave in the field of machine learning. LeCun [7] proposed the famous LeNet-5 model and applied for the first time CNN (Convoluted Neural Networks). However, no more attentions was paid on that due to the limitation of the computation capacity of the computer hardware. In 2012, the Alexnet model from Hinton team won two first places in the Imagenet competition[8], which attracted great attention of researchers. At the same time, deep learning showed great potential in remote sensing applications [9-11].

In this paper, the CNN was applied to classify the sea ice at the northern region of Liaodong Bay, Bohai, China, based on the optical image of Sentinel-2. The experimental results showed that the method proposed here have achieved better classification results compared with traditional classification algorithms.

\footnotetext{
*orresponding author: caihuiwen1977@hotmail.com
} 


\section{Materials and Methods}

\subsection{Study area}

Liaodong Bay is one of the three bays in the Bohai Sea, China, which starts from the Estuary of Daqing to the north of Laotieshan Cape in Liaodong peninsula. The submarine topography is inclined to the center from the top of the bay and the east and west sides. The water depth at the east side of the bay is higher than that at the west, with the deepest water of 32 meters. The sea temperature of Liaodong Bay is the lowest in all of the coastal area in China. There is the most severe sea ice conditions as well. Solid and thick sea ice happens every year which brings serious economy losses. The east coast is even worse than the west because of the strong northwest wind in winter. So, in this paper the eastern part of Liaodong Bay is selected as the study area with an area of $20 \mathrm{~km} \times 20 \mathrm{~km}$ (Figure 1).
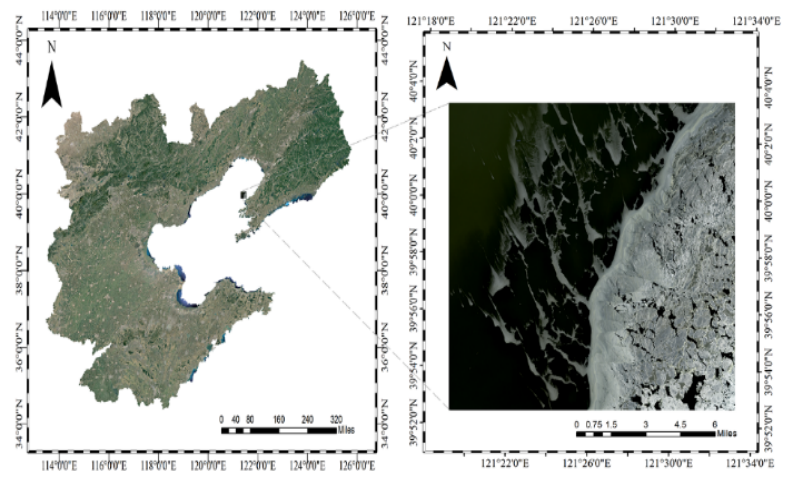

Fig.1. Location of the study area

\subsection{Experimental data}

In this paper, data from Sentinel-2 was used in the experiment, and the data time is on February, 2019. Sentinel-2 is the second satellite of ESA's global environment and safety monitoring program. It carries a multispectral imager which covers 13 spectral bands in a width of $290 \mathrm{~km}$. Sentinel-2 can provide the data with a high spatial resolution of 10 meters and revisit period of 10 days. Sentinel-2 covers a large light spectrum from visible light, near infrared to short wave infrared at different spatial resolution. Both the fast revisit period and the large image range all provide robust support for the sea ice monitoring. Sentinel-2 data can be downloaded at https://scihub.copernicus.eu/dhus/. The original image was manually annotated to obtain the data with labels so as to facilitate training of the convolutional neural network for sea ice analysis (Figure 2).

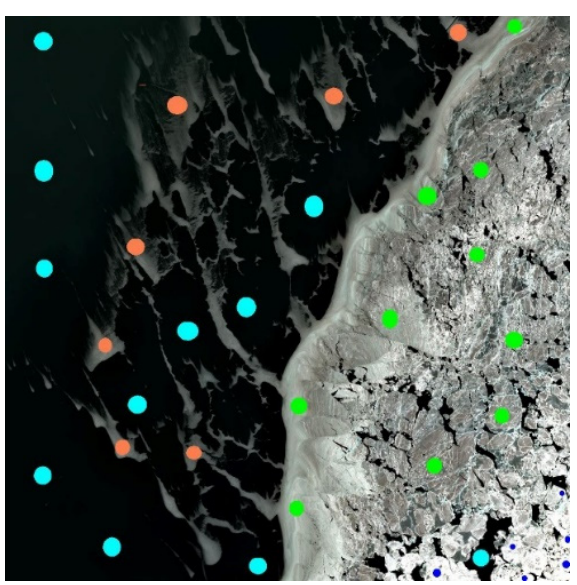

Fig.2. Labelled samples of study area

\subsection{Convolutional Neural Networks}

Convolutional Neural Network $(\mathrm{CNN})$ is a neural network which is particularly used to process data with similar grid structure [12]. CNN is composed of several convolutional layers, pooling layers and fully connected layers. The first several layers of $\mathrm{CNN}$ are composed of convolutional layers and pooling layers alternately, then the rest layers are all full connection layers[13]. Convolutional layer has the characteristics of strong learning ability to extract hierarchical features. ReLU (Rectified Linear Units) was used after convolutional layers Linear function as the activation function. The application of ReLU function can increase the training speed of neural network several times, and will not impact the accuracy of the model generalization significantly. The pooling layers can reduce the size of the model and improve the robustness of the extracted features. As a classifier, the fully connected layers take the features extracted from the convolutional layer as the input to map the learned distributed feature representation to the sample tag space. The structure of Convolutional Neural Network used in this paper is as shown in figure 3.

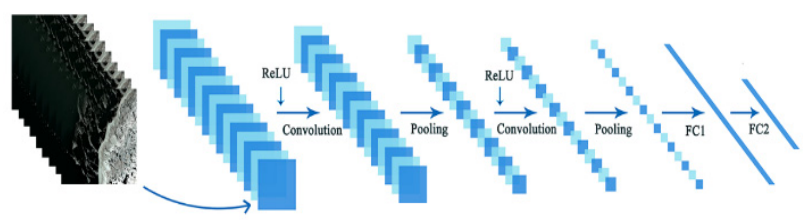

Fig.3. Structure of convolutional neural network

The process of two-dimensional convolutional operation can be expressed by formula (1). The first parameter of the convolution layer is usually called input(I), and the second parameter is called kernel function $(\mathrm{K})$, and the output is called feature map. In $\mathrm{CNN}$, the input is usually multi-dimensional array data, which is called tensor. The performance of convolution operation on multiple dimensions will be:

$S(i, j)=(I * K)(i, j)=\sum_{m} \sum_{n} I(m, n) K(i-m, j-n)$

Where,

$m, n$ is the size of input respectively and $i, j$ is the size of output. 
Pooling is essentially a form of down sampling in which the pooling function uses the overall statistical characteristics of adjacent elements at a location to replace the network output at that location. Generally, Max pooling is used to divide the images input into several rectangular areas, then got the maximum value for each sub-area, and finally output the original structure after discarding other nodes.

The purpose of the fully connected layer is to integrate the features extracted above, in which each neuron is fully connected with all neurons in the previous layers. The fully connected layers can integrate the local information with classification distinction in the convolution layer and pooling layer. The output of the last fully connected layer is classified by a softmax function.

\section{Experimental results and analysis}

The experimental hardware environment was Intel i5 $8500 \mathrm{CPU}$ 3.9GHz, NVIDIA GTX 1060 3GB GPU and 16GB RAM. The software environment was Python3.5 based TensorFlow, and NVIDIA CUDA 9.0 was used for GPU-accelerated calculation. In order to verify the effectiveness of the algorithm proposed in this paper, traditional methods such as the minimum distance method, maximum likelihood method, Mahalanobis distance method and support vector machine method were selected as the comparison algorithms. The overall accuracy and kappa coefficient are used as the accuracy measurement indicators. The experimental results of sea ice situations in Liaodong Bay are presented in Figure 4.
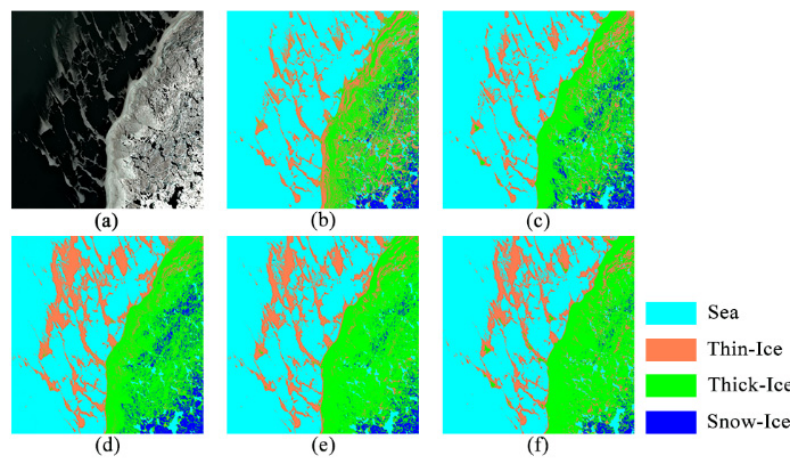

Fig.4. Classification results of sea ice. (a) false colour (b) minimum distance (c) maximum likelihood (d)

mahalanobis (e) support vector machine (f) CNN
From the sea ice images and the accuracy table (Table 1), we can see that for the sea water analysis, all of the algorithms can get a good results with an accuracy of over $80 \%$ (table 1). However, for the thin ice analysis, there were obvious leakage when analyzed by the minimum distance method and the maximum likelihood method. And some thick ice was misclassified as snowice. The reason may be because that the local sample in the study area is too small and it is hard for these methods to extract the more detailed features. The support vector machine method had a better classification results for thin ice and thick ice analysis. When snow-ice concentrated better, SVM also can achieve a good result. However, for small samples of thick ice and snow-ice, severe misclassification usually happened. In contrast, the convolutional neural network algorithm had a very accurate analysis on various categories of sea ice. The reason is that $\mathrm{CNN}$ has a very powerful feature extraction capability and can perform hierarchical feature extraction. The overall accuracy of CNN on sea ice analysis in this paper is $85.79 \%$, while the comparison method is $60.37 \%, 63.54 \%, 63.36 \%$, and $75.64 \%$ respectively.

\section{Conclusion}

A sea ice classification method based on convolution neural network $(\mathrm{CNN})$ is proposed in this paper. Convolutional neural network is a convolutional computation and has deep structure of feedforward neural networks. As one of the deep learning algorithms, $\mathrm{CNN}$ can extract the hierarchical feature of the input information, more layers of network enables CNN to extract the deeper characteristics of the provided information. The sea ice classification experiment in Liaodong Bay showed that CNN method in this paper had achieved remarkable results which deeply analyzed the high resolution and multi-spectral characteristics images of sea ice from Sentinel-2. The overall accuracy improved $25.42 \%, 22.25 \%, 22.43 \%$ and $10.15 \%$, respectively, compared with the four traditional classification methods. The extraction capability of CNN increases linearly with the depth of the network [14], However, the complexity the network structure will lead to more parameters calculation and increase the operation time. In the future, attentions should be focused on how to improve the computing efficiency and ensuring the accuracy at the same time. 
Table 1 Sea ice classification accuracy of the study area

\begin{tabular}{cccccc}
\hline Class & Minimum distance & Maximum likelihood & Mahalanobis distance & Support Vector Machine & CNN \\
\hline OA (\%) & 60.37 & 63.54 & 63.36 & 75.64 & 85.79 \\
Kappa (\%) & 58.62 & 61.89 & 62.25 & 69.33 & 84.51 \\
\hline Sea & 80.63 & 82.61 & 85.61 & 85.04 & 89.94 \\
Thin-Ice & 55.25 & 65.36 & 70.64 & 74.65 & 81.67 \\
Thick-Ice & 54.29 & 60.51 & 55.67 & 76.19 & 88.76 \\
Snow-Ice & 52.67 & 50.06 & 51.36 & 70.65 & 87.06 \\
\hline
\end{tabular}

\section{References}

1. Rigor I G, Wallace J M, Colony R L . Response of Sea Ice to the Arctic Oscillation. Journal of Climate. 15(18):2648-2663(2002)

2. Melling, Humfrey. Sound Scattering from Sea Ice: Aspects Relevant to Ice-Draft Profiling by Sonar. Journal of Atmospheric and Oceanic Technology. 15(4):1023-1034(1998)

3. Zhao W, Du S. Spectral-spatial feature extraction for hyperspectral image classification: A dimension reduction and deep learning approach[J]. IEEE Transactions on Geoscience and Remote Sensing, 54(8):4544-4554(2016)

4. DabboorM, Geldsetzer T. Towards sea ice classification using simulated RADARSAT Constellation Mission compact polarimetric SAR imagery. Remote Sensing of Environment.140:189195(2014)

5. Ressel R, Frost A, Lehner S . A Neural NetworkBased Classification for Sea Ice Types on X-Band SAR Images. IEEE Journal of Selected Topics in Applied Earth Observations and Remote Sensing. 8(7):1-9.(2015)

6. Ressel R, Singha S, Lehner S, et al. Investigation into Different Polarimetric Features for Sea Ice Classification Using X-Band Synthetic Aperture Radar. IEEE Journal of Selected Topics in Applied Earth Observations and Remote Sensing. 9(7):31313143(2016)

7. Lecun Y, Bottou L, Bengio Y, Haffner P. Gradientbased learning applied to document recognition. Proceedings of the IEEE, 86(11):2278-2324(1998)

8. Krizhevsky A, Sutskever I, Hinton G E. ImageNet Classification with Deep Convolutional Neural Networks. Neural Information Processing Systems, Curran Associates Inc (2012)

9. Chen Y S, Jiang H L, Li C Y, Jia X P and Ghamisi P. 2016. Deep Feature Extraction and Classification of Hyperspectral Images Based on Convolutional Neural Networks. IEEE Transactions on Geoscience and Remote Sensing, 54(10):6232-6251(2016)

10. Santara A, Mani K, Hatwar P, Singh A, Garg A, Padia K and Mitra P. 2017. BASS net: bandadaptive spectral-spatial feature learning neural metwork for hyperspectral image classification. IEEE Transactions on Geoscience and Remote Sensing, 55(9):5293-5301(2017)

11. Gong J Y, Ji S P. Photogrammetry and Deep Learning. Acta Geodaetica et Cartographica Sinica, 47(6): 693-704 (2018)

12. Goodfellow I, Bengio Y and Courville A. Deep Learning. Cambridge: Massachusetts Institute of Technology Press, 322-334(2016)

13. Raziye Hale Topaloğlu, Sertel E, Nebiye Musaoğlu. Assessment of classification accuracies of Sentinel2 and Landsat-8 data for land cover / use mapping. ISPR International Archives of the Photogrammetry, Remote Sensing and Spatial Information Sciences, XLI-B8:1055-1059(2016)

14. Shen $X$, Zhang J, Zhang $X$, et al. Sea Ice Classification Using Cryosat-2 Altimeter Data by Optimal Classifier-Feature Assembly. IEEE Geoscience and Remote Sensing Letters. 14(11):1948-1952(2017) 